\title{
Modelagem hidrológica em microbacia hidrográfica Parte I: Aprimoramento do modelo HidroBacia ${ }^{1}$
}

\author{
Sidney S. Zanetti ${ }^{2}$, José M. A. da Silva ${ }^{3}$, Elias F. Sousa ${ }^{4}$, Vicente de P. S. de O liveira $^{5}$ \& Frederico T. de Almeida ${ }^{4}$
}

\section{RESUMO}

Testes preliminares realizados com o model o hidrológico HidroBacia, indicaram desequilíbrio no seu balanço de massa, despertando a necessidade de estudos visando ao seu aperfeiçoamento; assim, o presente trabalho consistiu no aprimoramento do modelo HidroBacia a partir da local ização e correção de falhas no código-fonte do seu programa computacional, essas falhas foram identificadas através da depuração do modelo durante simulações do hidrograma de escoamento superficial, utilizandose dados de uma microbacia hidrográfica experimental. A principal causa relacionada com o desequilíbrio do balanço de massa do modelo, tinha referência com a solução das equações do modelo de ondas cinemáticas usado na propagação dos hidrogramas de escoamento superficial, cujo problema foi contornado pela substituição do algoritmo linear utilizado, Bras (1990), pelo al goritmo não-linear apresentado por Li etal. (1975). A partir desta modificação, considerada principal, e outras modificações implementadas, o balanço de massa do modelo HidroBacia foi ajustado e suas simulações passaram a gerar estimativas coerentes. Em virtude das alterações efetuadas, o HidroBacia evoluiu da versão 1.0 para a versão 1.1.

Palavras-chave: simulação hidrológica, computação, escoamento superficial, hidrologia, hidrogramas

\section{Hydrologic modeling in a small watershed Part I: Improvement of the HidroBacia model}

\begin{abstract}
In previous tests conducted by the HidroBacia hydrologic model, desequilibria in its mass balance was detected, thus showing the need for further studies for its improvement. This work consisted of improvement of the HidroBacia model by means of identification and correction of mistakes in its software source code. The mistakes were identified through adjustment during runoff hydrograph simulations using an experimental watershed data bank. The main factor, related to the mass balance desequilibria in the model, was correlated with the solution of the equations for the kinematic wave model used for propagation of the runoff hydrographs. This problem was corrected by the substitution of the linear algorithm used (Bras, 1990), by the non-linear algorithm proposed by Li et al. (1975). Starting from this modification, considered as the principal one, and other modifications made in the program, mass balance of the HidroBacia model was fitted and its simulations started to generate coherent estimates. Due to the modifications made in HidroBacia the software evolved from version 1.0 to version 1.1.
\end{abstract}

Key words: hydrologic simulation, computation, surface runoff, hydrology, hydrograph

\footnotetext{
1 Parte da tese de doutorado do primeiro autor, apresentada à Universidade Estadual do Norte Fluminense - UEN $F$

2 ID AF-ES. Rua Dom Fernando, n. 39, 2. andar, Bairro Independência, CEP 29306-350, Cachoeiro de Itapemirim, ES. Fone (28) 3517-3190. E-mail: ssz@bol.com.br

3 DEA/UFV. Viçosa, MG. CEP 36570-000 Viçosa, MG. Fone (31) 3899-1912. E-mail: jmarcio@gprh.ufv.br

${ }^{4}$ LEAG/UENF. CEP 28.013-602, Campos dos Goytacazes, RJ. Fone (22) 2726-1543. E-mail: efs@uenf.br; fredterr@uenf.br

${ }^{5}$ N PGA/CEFET. CEP 28030-130 Campos dos Goytacazes, RJ. Fone (22) 2726-2800. E-mail: vsantos@cefetcampos.br
} 


\section{INTRODUÇÃO}

A compreensão sobre os processos hidrológicos, é fundamental em estudos ambientais, na gestão dos recursos hídricos e em projetos de obras hidráulicas. O tempo em que a água permanece nas diversas partes da hidrosfera influencia, entre outros, a disponibilidade hídrica, a ocorrência de inundações e a dinâmica de elementos, nutrientes e poluentes. A importância dessas inter-relações é responsável pelo crescente interesse em estudos hidrológicos; neste contexto, a modelagem hidrológica é utilizada como ferramenta para se obter conhecimento mais aprofundado a respeito dos fenômenos físicos envolvidos e na previsão de cenários (Moraes, 2003).

Uma das grandes dificuldades para o planejamento adequado e o manejo integrado dos recursos hídricos, diz respeito à falta de métodos que permitam estimar o efeito dos diversos fatores que interferem no processo de produção de escoamento superficial, tendo em vista o fato de que os métodos desenvolvidos no exterior apresentam limitações quanto ao uso para as condições edafoclimáticas brasileiras.

Visando obter um modelo mais representativo das condições brasileiras, Silva (1999) desenvolveu um método e um programa computacional denominado Hidrograma. A partir de um evento de precipitação o modelo permite obter o hidrograma de escoamento superficial em qualquer posição de uma encosta regular (declividade constante), para solos com umidade próxima à saturação (condição extrema de ocorrência do escoamento superficial) e para seções transversais de canais de terraços ou drenos de superfície.

Ao aperfeiçoar o modelo Hidrograma, Braga (2000) desenvolveu um novo programa computacional, denominado HidroBacia; trata-se de um modelo com parâmetros distribuídos, viabilizado pela discretização da bacia hidrográfica em unidades de simulação (células) uniformes e quadradas, através de sistemas de informações geográficas (SIG).

O modelo HidroBacia possibilita a estimação do hidrograma de escoamento superficial em qualquer posição de uma bacia hidrográfica (declividade variada), mesmo para condições de umidade diferentes da saturação. Para obtenção do hidrograma na célula de interesse, o hidrograma nela produzido é somado com os hidrogramas advindos das células contidas na sua área de contribuição. Na identificação das células contribuintes, Braga (2000) considerou apenas as quatro células vizinhas ortogonais, desprezando as células vizinhas diagonais.

Além desses aperfeiçoamentos, Braga (2000) promoveu, também, a substituição do componente relativo às abstrações iniciais da precipitação pelos processos de interceptação pela cobertura vegetal e armazenamento superficial.

Com base no método do balanço hídrico proposto por Pruski et al. (2001), Silva (2002) aperfeiçoou o modelo HidroBacia possibilitando, além da obtenção do hidrograma de escoamento superficial, a realização do balanço hídrico, considerando os processos de precipitação, evapotranspiração, interceptação pela cobertura vegetal, armazenamento superficial, infiltração da água no solo e escoamento superficial.

Na modelagem do escoamento superficial Silva (2002), adotou o modelo de ondas cinemáticas para a propagação dos hidrogramas. O hidrograma resultante de cada célula continuou sendo obtido pela soma do hidrograma produzido na própria célula com os hidrogramas advindos da sua área de contribuição, mas na identificação das células contribuintes foram consideradas as oito células vizinhas, ortogonais e diagonais, conforme proposto no algoritmo Deterministic-8Node (O'Callaghan \& Mark, 1984).

Silva (2002) aprimorou e testou o modelo HidroBacia utilizando uma microbacia hidrográfica com cobertura vegetal homogênea e considerando uniformes os parâmetros de entrada da bacia usados do modelo. $\mathrm{O}$ autor ressaltou, ainda, a necessidade de continuação dos estudos sobre o HidroBacia e recomendou a realização de testes, tendo em vista a variabilidade espacial dos parâmetros.

$\mathrm{O}$ presente trabalho surgiu do interesse de se utilizar o modelo hidrológico HidroBacia, aliado à necessidade de continuação dos estudos sobre o mesmo, conforme sugerido por Silva (2002); além disso, testes preliminares realizados com o modelo indicaram desequilíbrio no seu balanço de massa, ressaltando ainda mais a necessidade do seu aperfeiçoamento.

Ante o exposto, desenvolveu-se este trabalho com o objetivo de aprimorar o modelo hidrológico HidroBacia por meio de simulações do balanço hídrico e do hidrograma de escoamento superficial em uma microbacia hidrográfica experimental, ressaltando-se a variabilidade espacial dos parâmetros de entrada da microbacia associados ao processo de produção de escoamento superficial.

\section{MATERIAL E MÉTODOS}

O aprimoramento do modelo HidroBacia foi realizado a partir da localização e correção de falhas no código-fonte do seu programa computacional. Essas falhas foram identificadas em simulações do escoamento superficial, utilizando-se dados de uma microbacia hidrográfica experimental, com área de 88,5 ha, localizada na Bacia do Rio Paraíba do Sul, município de Varre-Sai, noroeste do Estado do Rio de Janeiro.

A microbacia utilizada possui diversos tipos de cobertura vegetal, incluindo-se áreas com mata atlântica, eucalipto, pastagem, cafeicultura, culturas anuais e vegetação espontânea em estágio inicial de regeneração; possui, ainda, várias nascentes que formam e mantêm um pequeno curso d'água perene.

De início, realizou-se o levantamento dos dados de entrada referentes à cobertura vegetal e às características físicas do solo da microbacia, os quais foram inseridos no modelo, na forma de imagens temáticas, considerando-se sua distribuição espacial; para tanto, a microbacia foi discretizada em unidades de simulação (células) uniformes e quadradas, com cinco metros de lado, dispostas em linhas e colunas, com técnicas de sistema de informações geográficas (SIG). O modelo necessitou, ainda, das imagens relativas ao modelo digital de elevação do terreno (MDE), às direções de escoamento e à rede de drenagem numérica (canais), para cuja obtenção se realizou, também, o levantamento topográfico da microba- 
cia, sendo os dados processados através de programas computacionais baseados em SIG.

Nas simulações com o HidroBacia a precipitação foi fornecida na forma de pluviogramas, os quais foram registrados usando-se uma estação meteorológica automática (Adolf Thies GmbH Co., Alemanha) instalada na microbacia.

Na modelagem hidrológica o HidroBacia considera que, ao se iniciar a precipitação, começa também, simultaneamente, a interceptação pela cobertura vegetal (ICV, mm), que representa a fração da precipitação retida pela vegetação. Após o preenchimento da ICV a água atinge o solo, elevando sua umidade e diminuindo a capacidade de infiltração. Quando a intensidade de precipitação supera a capacidade de infiltração da água no solo, a água acumula em sua superfície. Uma vez que a capacidade de armazenamento superficial (ARM, mm) é excedida, tem-se o início do escoamento superficial.

Conforme proposto por Silva (2002), os hidrogramas oriundos das células que contribuem com o escoamento superficial são obtidos com o modelo de ondas cinemáticas, uma das formas de aplicação das equações de Saint-Venant (Tucci, 1998), que pode ser expresso por:

$$
\frac{\partial \mathrm{q}}{\partial \mathrm{x}}+\frac{\partial \mathrm{h}}{\partial \mathrm{t}}=\mathrm{i}_{\mathrm{i}}-\mathrm{T}_{\mathrm{i}}
$$

em que:

q - vazão por unidade de largura na direção do escoamento, $\mathrm{m}^{2} \mathrm{~s}^{-1}$

$\mathrm{x}$ - direção do escoamento, $\mathrm{m}$

h - lâmina de escoamento, $\mathrm{m}$

$\mathrm{t}$ - tempo, $\mathrm{s}$

$\mathrm{i}_{\mathrm{i}}$ - intensidade de precipitação, $\mathrm{mm} \mathrm{h}^{-1}$

$\mathrm{T}_{\mathrm{i}}$ - taxa de infiltração de água no solo, $\mathrm{mm} \mathrm{h}^{-1}$, calculada usando-se a equação de Green-Ampt modificada por Mein \& Larson (1973)

O modelo de ondas cinemáticas considera a declividade da linha de energia igual à declividade da superfície do solo $\left(\mathrm{S}_{0}, \mathrm{~m} \mathrm{~m}^{-1}\right)$ assumindo, assim, uma seção transversal média de escoamento. Com a utilização de equações normalmente usadas para escoamento em condições de regime uniforme, obtêm-se as relações entre a vazão e a profundidade de escoamento, expressas por:

$$
h=\alpha q^{\beta}
$$

Os parâmetros $\alpha$ e $\beta$ são obtidos a partir da equação de Manning, cujos valores dependem da declividade do canal (ou encosta) e da rugosidade do perímetro molhado, podendo ser expressos por:

$$
\alpha=\left(\frac{\mathrm{n}}{\sqrt{\mathrm{S}_{0}}}\right)^{\beta} \quad \beta=\frac{3}{5}
$$

em que $\mathrm{n}$ é o coeficiente de rugosidade do terreno, $\mathrm{s} \mathrm{m}^{-1 / 3}$.

A declividade $\mathrm{S}_{0}$ é calculada com base na cota da célula vizinha, para a qual ocorre o escoamento superficial, por meio da equação:

$$
\mathrm{S}_{0}=\frac{\mathrm{Z}_{1}-\mathrm{Z}_{2}}{\mathrm{D}_{12}}
$$

em que:

$\mathrm{Z}_{1}$ - cota da célula considerada, $\mathrm{m}$

$\mathrm{Z}_{2}$ - cota da célula para a qual ocorre escoamento, $\mathrm{m}$

$D_{12}$ - distância entre os centros das células consideradas, $m$

A modelagem do escoamento superficial no canal também é realizada utilizando-se o modelo de ondas cinemáticas, caso em que o escoamento superficial é quantificado pela soma dos hidrogramas advindos dos segmentos do canal à montante da seção considerada com os hidrogramas oriundos da encosta.

O desequilíbrio do balanço de massa do modelo HidroBacia foi identificado analisando-se a equação seguinte:

$$
\mathrm{PT}-(\mathrm{ICV}+\mathrm{ARM}+\mathrm{LI}+\mathrm{LES})=0
$$

em que:
PT - precipitação total, mm
U - lâmina infiltrada, mm
LES - lâmina de escoamento superficial, mm

A condição apresentada na Eq. 5 não estava sendo atendida na modelagem hidrológica, infringindo o princípio básico da conservação de massa; a partir desta constatação, o aprimoramento do modelo foi orientado visando anular o seu balanço de massa e se adotando a superfície do solo como referência. Na busca pelas causas do desequilíbrio várias correções foram implementadas, inclusive as não relacionadas com o referido problema, que também foram identificadas.

Em virtude da utilização de eventos de precipitação isolados nas simulações, a evapotranspiração não foi considerada no balanço de massa, uma vez que a lâmina evapotranspirada durante esses eventos é muito pequena devido à curta duração dos mesmos, além das condições de céu nublado e alta umidade relativa do ar.

$\mathrm{Na}$ busca pelo equilíbrio entre as entradas e saídas do modelo HidroBacia, a primeira causa identificada estava relacionada com o algoritmo linear proposto por Bras (1990), utilizado por Silva (2002) para solucionar as equações do modelo de ondas cinemáticas; tal algoritmo estava produzindo hidrogramas com volumes diferentes daqueles observados nos hidrogramas iniciais desequilibrando, assim, o balanço de massa do modelo, fato que despertou a necessidade de substituição do referido algoritmo; então, após uma pesquisa bibliográfica se optou por utilizar a solução não-linear apresentada por Li et al. (1975), sendo esta a principal alteração proposta para o modelo HidroBacia, descrita na seqüência.

Representando a Eq. 1 e se utilizando o método das diferenças finitas aplicado ao plano espaço-tempo apresentado na Figura 1, obtêm-se a equação:

$$
\frac{\mathrm{q}_{\mathrm{i}+1}^{\mathrm{j}+1}-\mathrm{q}_{\mathrm{i}}^{\mathrm{j}+1}}{\Delta \mathrm{x}}+\frac{\mathrm{h}_{\mathrm{i}+1}^{\mathrm{j}+1}-\mathrm{h}_{\mathrm{i}+1}^{\mathrm{j}}}{\Delta \mathrm{t}}=\mathrm{i}_{\mathrm{i}}-\mathrm{T}_{\mathrm{i}}
$$

Ao contrário de Bras (1990), no desenvolvimento da solução, Li et al. (1975) tomaram q como variável independente na Eq. 2; representando, também, esta equação na forma de 


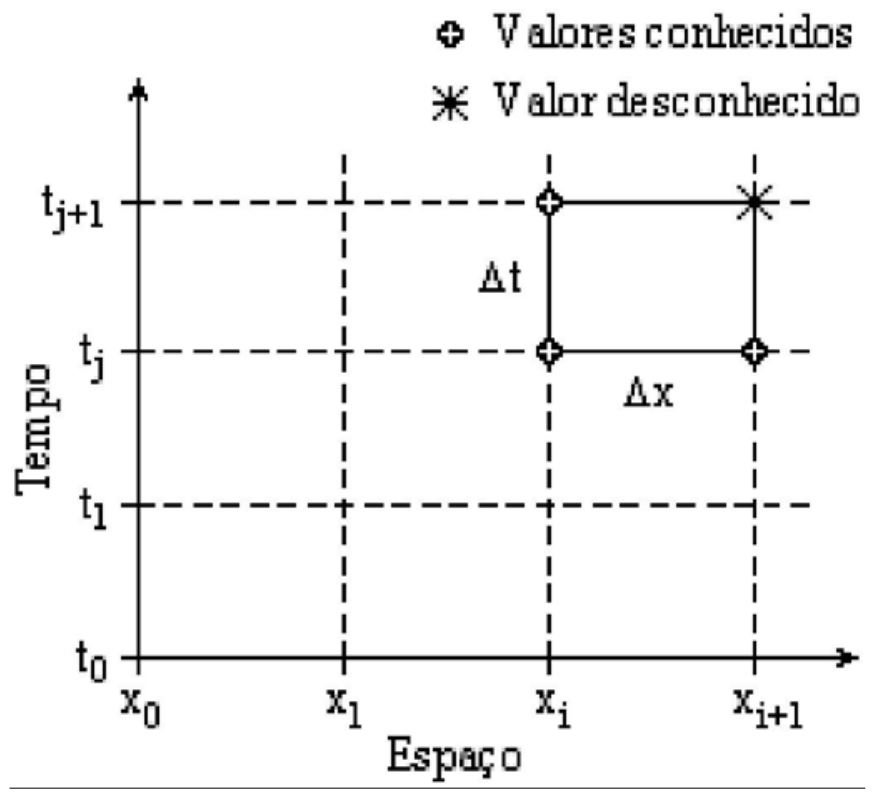

Figura 1. Plano espaço-tempo utilizado para ilustrar a solução das equações do modelo de ondas cinemátic as usando-se diferenças finitas

diferenças finitas, substituindo-a na Eq. 6 e, rearranjando-a, obtém-se:

$$
\frac{\Delta t}{\Delta x} q_{i+1}^{j+1}+\alpha\left(q_{i+1}^{j+1}\right)^{\beta}=\frac{\Delta t}{\Delta x} q_{i}^{j+1}+\alpha\left(q_{i+1}^{j}\right)^{\beta}+\Delta t\left(i_{i}-T_{i}\right)
$$

O lado direito da Eq. 7 possui apenas quantidades conhecidas e pode ser denotado por $\Omega$. Fazendo $\mathrm{q}_{\mathrm{i}+1}^{\mathrm{j}+1}=\mathrm{r}$, a Eq. 7 pode ser expressa por:

$$
f(r)=\frac{\Delta t}{\Delta x} r+\alpha r^{\beta}=\Omega
$$

A resolução do problema consiste em encontrar o valor de $r$ que satisfaça a Eq. 8; como esta equação é não-linear em r, uma solução aproximada para a mesma pode ser obtida com o seguinte esquema iterativo, apresentado por Li et al. (1975):

$$
r^{k+1}=r^{k}-\frac{f^{\prime}\left(r^{k}\right)}{f^{\prime \prime}\left(r^{k}\right)} \pm \sqrt{\left(\frac{f^{\prime}\left(r^{k}\right)}{f^{\prime \prime}\left(r^{k}\right)}\right)^{2}-\frac{2\left[f\left(r^{k}\right)-\Omega\right]}{f^{\prime \prime}\left(r^{k}\right)}}
$$

em que $\mathrm{r}^{\mathrm{k}}$ representa o valor de $\mathrm{r}$ na k-ésima iteração; $\mathrm{f}^{\prime}$ e f" são, respectivamente, as primeira e segunda derivadas da função:

$$
\mathrm{f}\left(\mathrm{r}^{\mathrm{k}}\right)=\frac{\Delta \mathrm{t}}{\Delta \mathrm{x}} \mathrm{r}^{\mathrm{k}}+\alpha\left(\mathrm{r}^{\mathrm{k}}\right)^{\beta}
$$

As iterações são realizadas até que $\left|\mathrm{f}\left(\mathrm{r}^{\mathrm{k}+1}\right)-\Omega\right|$ seja um valor próximo de zero $(\varepsilon)$. Segundo Li et al. (1975), um valor apropriado para $\varepsilon$ é $0,01 \Omega$; entretanto, preferiu-se utilizar um valor fixo, $\varepsilon=10^{-9}$; assim, o critério de parada adotado foi $\left|\mathrm{f}\left(\mathrm{r}^{\mathrm{k}+1}\right)-\Omega\right|$ $\leq \varepsilon$; como se observa, a Eq. 9 possui duas soluções, sendo escolhida a que apresentar o menor valor de $\left|\mathrm{f}\left(\mathrm{r}^{\mathrm{k}+1}\right)-\Omega\right|$.
De acordo com Li et al. (1975), uma boa estimativa do valor inicial $\mathrm{r}^{0}$ é determinante para uma rápida convergência do processo iterativo indicando-se, para isto, a solução linear descrita a seguir.

$\mathrm{O}$ termo $\partial \mathrm{h} / \partial \mathrm{t}$ da Eq. 1 pode ser expresso por:

$$
\frac{\partial \mathrm{h}}{\partial \mathrm{t}}=\frac{\partial \mathrm{h}}{\partial \mathrm{q}} \frac{\partial \mathrm{q}}{\partial \mathrm{t}}
$$

Derivando a Eq. 2 ( $\partial \mathrm{h} / \partial \mathrm{q})$, substituindo-a na Eq. 11 e substituindo a equação resultante no termo $\partial \mathrm{h} / \partial \mathrm{t}$ da Eq. 1, resulta em:

$$
\frac{\partial \mathrm{q}}{\partial \mathrm{x}}+\alpha \beta \mathrm{q}^{(\beta-1)} \frac{\partial \mathrm{q}}{\partial \mathrm{t}}=\mathrm{i}_{\mathrm{i}}-\mathrm{T}_{\mathrm{i}}
$$

Representando-se a Eq. 12 na forma de diferenças finitas e rearranjando-a, fica:

$$
r^{0}=q_{i+1}^{j+1}=\frac{\frac{\Delta t}{\Delta x} q_{i}^{j+1}+\alpha \beta q_{i+1}^{j}\left(\frac{q_{i+1}^{j}+q_{i}^{j+1}}{2}\right)^{\beta-1}+\Delta t\left(i_{i}-T_{i}\right)}{\frac{\Delta t}{\Delta x}+\alpha \beta\left(\frac{q_{i+1}^{j}+q_{i}^{j+1}}{2}\right)^{\beta-1}}
$$

A Eq. 13 permite obter a melhor estimativa de $\mathrm{r}^{0}$ usado como valor inicial para o esquema não-linear iterativo representado pela Eq. 9; a resolução desta equação para todos os pontos do hidrograma, promove o deslocamento do mesmo para a célula que receberá o escoamento. Uma vez que o hidrograma é deslocado, pode-se realizar a sua soma com o hidrograma gerado na célula que receberá o escoamento.

A precisão das estimativas da Eq. 9 pode ser melhorada diminuindo-se os valores de $\Delta \mathrm{x}$ e $\Delta \mathrm{t}$. Utilizou-se, no presente trabalho, $\Delta \mathrm{t}=12 \mathrm{~s}$, sendo que o valor de $\Delta \mathrm{x}$ variou em função do tamanho das células no MDE e da direção do escoamento; como a direção de escoamento foi perpendicular ao lado da célula, utilizou-se $\Delta \mathrm{x}=5,0 \mathrm{~m}$ e, onde foi diagonal, utilizou-se $\Delta \mathrm{x}=7,071 \mathrm{~m}$.

\section{RESULTADOS E DISCUSSÃO}

O desequilíbrio apresentado no balanço de massa do modelo HidroBacia despertou dúvidas sobre a validade dos resultados até então gerados, com o seu uso. Estudos realizados com o modelo, como a análise de sensibilidade apresentada por Zanetti et al. (2005), certamente perderam a confiabilidade.

A alteração no método de solução das equações do modelo de ondas cinemáticas implicou na alteração da vazão máxima de escoamento superficial simulada e no seu tempo de ocorrência (tempo de concentração) vez que, durante a propagação dos hidrogramas de escoamento superficial, ocorre uma redistribuição das vazões ao longo do tempo, ou seja, o modelo de ondas cinemáticas promove o retardamento e a atenuação da onda de escoamento, considerando-se o efeito da declividade $\left(\mathrm{S}_{0}\right)$ e da rugosidade do terreno $(\mathrm{n})$; contudo, 
o volume de escoamento é preservado, atendendo ao princípio da conservação da massa.

Um exemplo de hidrograma propagado pelo modelo de ondas cinemáticas usando-se a solução não-linear apresentada por Li et al. (1975), pode ser observado na Figura 2. Ressalta-se que o volume de escoamento superficial do hidrograma propagado não deve diferir do hidrograma inicial, conforme ocorreu neste exemplo e nos demais hidrogramas testados, usando-se o HidroBacia, após a substituição do método de resolução das equações do modelo de ondas cinemáticas; portanto, o algoritmo proposto por Li et al. (1975) apresentou resultados satisfatórios ao ser utilizado no modelo HidroBacia.

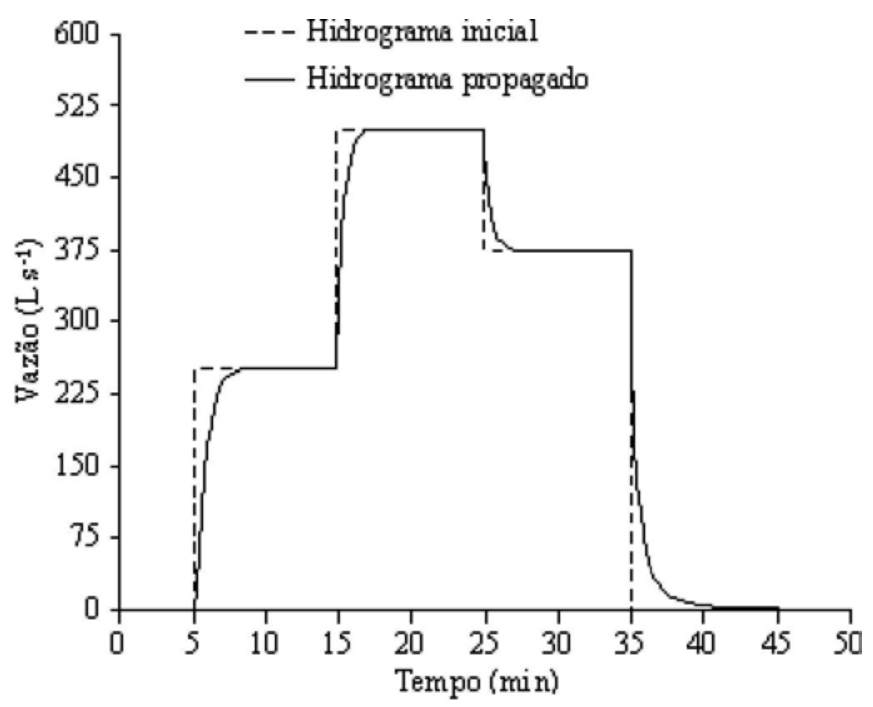

Figura 2. Exemplo de propagação de hidrograma pelo modelo de ondas cinemátic as, usando-se a solução não-linear apresentada por Li etal. (1975)

Mesmo após esta significativa alteração na modelagem hidrológica, o HidroBacia continuou apresentando balanço de massa desequilibrado em suas simulações.

Analisando-se o código-fonte do programa computacional HidroBacia, verificou-se que no seu desenvolvimento vários aspectos passaram despercebidos, provavelmente porque Silva (2002), na fase de teste do modelo, considerou homogênea a microbacia hidrográfica empregada, ou seja, desconsiderou a variabilidade espacial dos dados de entrada referentes ao solo e à cobertura vegetal da bacia.

Desta forma, utilizando-se os referidos dados de entrada na forma de imagens (mapas digitais considerando a variação espacial) e tendo como referência a correção do balanço de massa do modelo, várias falhas foram localizadas e corrigidas, algumas das quais estavam relacionadas com a utilização inadequada de comparações entre números reais para a tomada de decisões na modelagem, ou seja, a presença de pequenos erros comumente gerados no processamento numérico computacional estava gerando diferenças entre variáveis que deveriam ser iguais em determinados momentos, devido ao critério de comparação direta utilizado entre as mesmas, casos em que o problema foi contornado passandose a considerar duas variáveis reais iguais quando o módulo da diferença entre elas for um valor suficientemente pequeno, isto é, diferença desprezível.

A partir dessas e outras modificações implementadas no código-fonte, o balanço de massa do HidroBacia foi corrigido e as simulações passaram a gerar estimativas coerentes. $\mathrm{O}$ balanço de massa do modelo foi monitorado em mais de 500 simulações, sendo que em todas o comportamento foi satisfatório consolidando, assim, a resolução do problema. Em virtude das alterações efetuadas no modelo e no seu programa computacional, o HidroBacia evoluiu da versão 1.0 para a versão 1.1.

Apresentam-se, na Figura 3, três hidrogramas de escoamento superficial simulados pelo HidroBacia a partir de um evento de precipitação intensa, visando testar o seu comportamento; observa-se que os hidrogramas simulados apresentaram dois picos de vazão para uma precipitação com pico único; este comportamento surgiu em virtude do modelo considerar que diferentes hidrogramas são gerados para as diferentes áreas da bacia hidrográfica, como normalmente ocorre em condições reais, considerando-se precipitação uniforme em toda a bacia, porém os hidrogramas que o HidroBacia simulou para as diferentes áreas da microbacia foram mais discrepantes entre si do que os hidrogramas que comumente são produzidos em condições reais; em conseqüência, na soma desses diferentes hidrogramas dois picos foram gerados, demonstrando os hidrogramas predominantes em cada situação.

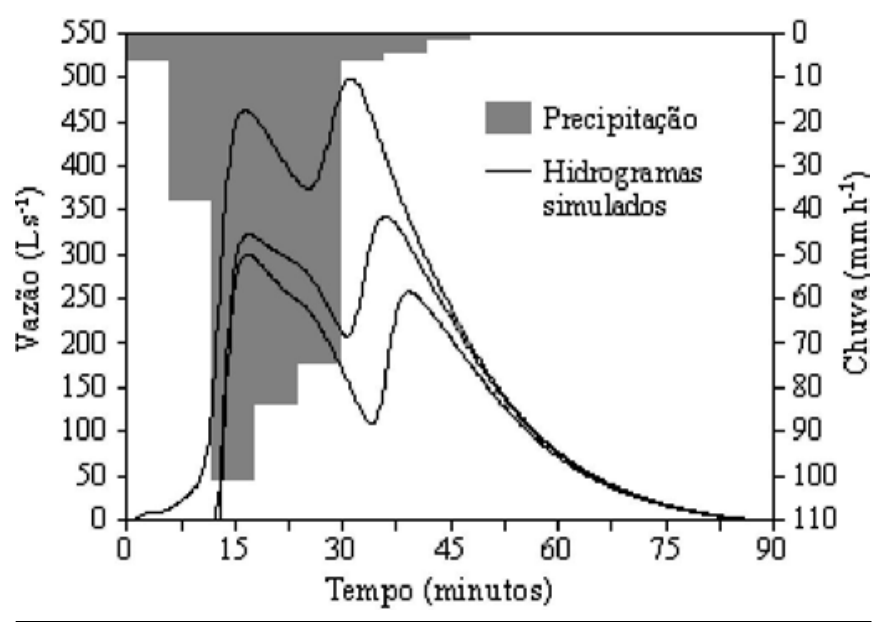

Figura 3. Hidrogramas de escoamento superficial simulados pelo HidroBacia utilizando-se um evento de precipitação intensa

Essas discrepâncias entre hidrogramas gerados em diferentes áreas da microbacia, resultam das diferenças que as mesmas apresentam nos valores de interceptação pela cobertura vegetal, armazenamento superficial e coeficiente de rugosidade do terreno, além da influência produzida pela discretização da microbacia em células, por meio do modelo digital de elevação. Enquanto a interceptação pela cobertura vegetal e $o$ armazenamento superficial influenciam no tempo de início do escoamento superficial, a rugosidade do terreno e o gradiente de declividade modificam a propagação dos hidrogramas, representada pelo modelo de ondas cinemáticas. 
Ressalta-se que os valores de interceptação pela cobertura vegetal, armazenamento superficial e coeficiente de rugosidade do terreno, utilizados no presente trabalho, não foram medidos na microbacia, mas foram obtidos a partir do banco de dados incluído no programa HidroBacia; portanto, é provável que tais valores não representem adequadamente as condições reais da microbacia hidrográfica experimental proporcionando, assim, as discrepâncias supracitadas.

\section{CONCLUSÕES}

1. O balanço de massa do modelo HidroBacia demandou correções; portanto, várias alterações foram implementadas no código-fonte do programa computacional, dentre elas a substituição do método utilizado para a solução das equações do modelo de ondas cinemáticas, passando-se a utilizar o algoritmo apresentado por Li et al. (1975).

2. Em virtude das alterações efetuadas no modelo e no seu programa computacional, o HidroBacia evoluiu da versão 1.0 para a versão 1.1.

\section{LITERATURA CITADA}

Braga, J. C. Modelo hidrológico de parâmetros distribuídos para obtenção do hidrograma de escoamento superficial. Viçosa: UFV, 2000. 76p. Dissertação Mestrado

Bras, R. L. Hydrology: An introduction to hydrological sciences. New York: Addison-Wesley, 1990. 643p.
Li, R. M.; Simons, D. B.; Stevens, M. A. Nonlinear kinematic wave approximation for water routing. Water Resources Research, v.11, n.2, p.245-252, 1975.

Mein, R. G.; Larson, C. L. Modeling infiltration during a steady rain. Water Resources Research, v.9, n.4, p.384-394, 1973.

Moraes, J. M. Propriedades físicas dos solos na parametrização de um modelo hidrológico. Revista Brasileira de Recursos Hídricos, v.8, n.1, p.61-70, 2003.

O'Callaghan, J. F.; Mark, D. M. The extraction of drainage networks from digital elevation data. Computer Vision, Graphics and Image Processing, v.28, p.323-344, 1984.

Pruski, F. F.; Rodrigues, L. N.; Silva, D. D. Modelo hidrológico para estimar o escoamento superficial em áreas agrícolas. Revista Brasileira de Engenharia Agrícola e Ambiental, v.5, n.2, p.301-307, 2001.

Silva, J. M. A. Metodologia para obtenção do hidrograma de escoamento superficial para qualquer ponto ao longo de uma encosta. Viçosa: UFV, 1999. 61p. Dissertação Mestrado

Silva, J. M. A. Modelo hidrológico para o cálculo do balanço hídrico e obtenção do hidrograma de escoamento superficial em bacias hidrográficas: Desenvolvimento e aplicação. Viçosa: UFV, 2002. 142p. Tese Doutorado

Tucci, C. E. M. Modelos hidrológicos. Porto Alegre: UFRGS/ ABRH, 1998. 669p.

Zanetti, S. S.; Oliveira, V. P. S.; Sousa, E. F.; Silva, J. M. A.; Almeida, F. T. Análise de sensibilidade do modelo HidroBacia. In: Congresso Brasileiro de Engenharia Agrícola, 34, 2005, Canoas. Resumos... Canoas: SBEA, 2005. CD Rom 